\title{
Cannabis Sativa: uma revisão integrativa dos aspectos legais, toxicológicos e
}

\section{farmacoterapêuticos}

\author{
Cannabis Sativa: an integrative review of legal, toxicological and pharmacotherapeutic aspects
}

Cannabis Sativa: una revisión integradora de los aspectos legales, toxicológicos y

farmacoterapéuticos

Recebido: 27/10/2021 | Revisado: 05/11/2021 | Aceito: 10/11/2021 | Publicado: 15/11/2021

\author{
Daniele Oliveira Cabral Pessoa \\ ORCID: https://orcid.org/0000-0001-5565-435X \\ Centro Universitário Unifavip Wyden, Brasil \\ E-mail: danielecabral488@gmail.com \\ Iago Vilar Lira \\ ORCID: https://orcid.org/0000-0002-5696-0348 \\ Centro Universitário Unifavip Wyden, Brasil \\ E-mail: ago_vilar@hotmail.com \\ Lidiany da Paixão Siqueira \\ ORCID: https://orcid.org/0000-0002-9742-2642 \\ Centro Universitário Unifavip Wyden, Brasil \\ E-mail: Lidianypaixao.farmacia@gmail.com
}

\begin{abstract}
Resumo
Atualmente, com o crescente número de estudos envolvendo fitocompostos dotados de inúmeras aplicações farmacológicas, a Cannabis passou a assumir um papel de protagonismo no tratamento de inúmeras patologias. Nesse contexto o delta 9-tetraidrocanabinol ( $\triangle 9$-THC), o canabidiol (CBD) e o canabinol (CBN), presentes principalmente no gênero Cannabis tem ganhado um maior enfoque científico como fonte natural desses compostos, que comparados a outras susbtâncias farmacológicas, apresentam um menor número de efeitos adversos. A pesquisa por evidências foi realizada nas bases de dados Medline, PubMed, Scielo e LILACS. Foram selecionados quarenta e um artigos que atenderam aos critérios de inclusão e de qualidade. Observou-se que a literatura científica apresenta fortes evidências do emprego dessas substâncias no tratamento de náuseas e vómitos associados à quimioterapia, analgesia na dor crónica, anorexia associada à Síndrome da Imunodeficiência Adquirida (AIDS), dor neuropática e algumas doenças neurológicas como a esclerose múltipla, ansiedade, insónia, epilepsia e na doença de Parkinson. Conclui-se que o uso dos fitocanabinoides apresentou um perfil toxicológico seguro para uso, tanto em crianças como em adultos, não estando relacionados com desenvolvimento de problemas secundários consideráveis ou dependência, sendo crescente sua aplicabilidade em fórmulas farmacêuticas na área industrial.
\end{abstract}

Palavras-chave: Fitocanabinoides; Cannabis sativa; Sistema nervoso central; Patologias.

\begin{abstract}
Currently, with the growing number of studies involving phytocompounds with numerous pharmacological applications, cannabis has taken a leading role in the treatment of numerous pathologies. In this context, delta 9tetrahydrocannabinol ( $\triangle 9$-THC), cannabidiol (CBD), and cannabinol (CBN), present mainly in the Cannabis genus, have gained greater scientific focus as a natural source of these compounds, which, compared to other pharmacological substances, have fewer adverse effects. The search for evidence was conducted in the Medline, PubMed, Scielo, and LILACS databases. Forty-one articles that met the inclusion and quality criteria were selected. It was observed that the scientific literature presents strong evidence for the use of these substances in the treatment of nausea and vomiting associated with chemotherapy, analgesia in chronic pain, anorexia associated with Acquired Immune Deficiency Syndrome (AIDS), neuropathic pain and some neurological diseases such as multiple sclerosis, anxiety, insomnia, epilepsy, and in Parkinson's disease. It is concluded that the use of phytocannabinoids presented a safe toxicological profile for use in both children and adults, not being related to the development of considerable secondary problems or dependence, and their applicability in pharmaceutical formulations in the industrial area is increasing.
\end{abstract}

Keywords: Phytocannabinoids; Cannabis sativa; Central nervous system; Pathologies.

\section{Resumen}

En la actualidad, con el creciente número de estudios sobre fitocompuestos con numerosas aplicaciones farmacológicas, el cannabis ha adquirido un papel destacado en el tratamiento de numerosas enfermedades. En este contexto, el delta 9-tetrahidrocannabinol ( $\triangle 9$-THC), el cannabidiol (CBD) y el cannabinol (CBN), presentes 
principalmente en el género Cannabis, han cobrado mayor protagonismo científico como fuente natural de estos compuestos que, en comparación con otras sustancias farmacológicas, tienen menos efectos adversos. La búsqueda de evidencias se realizó en las bases de datos Medline, PubMed, Scielo y LILACS. Se seleccionaron 41 artículos que cumplían los criterios de inclusión y calidad. Se observó que la literatura científica presenta fuertes evidencias del empleo de estas sustancias en el tratamiento de las náuseas y vómitos asociados a la quimioterapia, la analgesia en el dolor crónico, la anorexia asociada al Síndrome de Inmunodeficiencia Adquirida (SIDA), el dolor neuropático y algunas enfermedades neurológicas como la esclerosis múltiple, la ansiedad, el insomnio, la epilepsia y en la enfermedad de Parkinson. Se concluye que el uso de fitocannabinoides presentó un perfil toxicológico seguro para su uso, tanto en niños como en adultos, no estando relacionado con el desarrollo de problemas secundarios considerables o de dependencia, y su aplicabilidad en fórmulas farmacéuticas en el área industrial es cada vez mayor.

Palabras clave: Fitocannabinoides; Cannabis sativa; Sistema nervioso central; Patologías.

\section{Introdução}

A Cannabis, popularmente conhecida como maconha, é uma planta pertencente à família cannabaceae, que abrange três subtipos: Cannabis ruderalis, Cannabis indica e a Cannabis sativa, sendo a última considerada a terceira mais consumida em todo o mundo, representando a substância recreativa ilícita mais utilizada nacionalmente (HALL; WEIER, 2015). É uma planta que vem sendo utilizada há séculos pela humanidade para diversos fins, como alimentação, rituais religiosos, artifícios medicinais e para a produção de tecidos, já que a sua plantação produz mais fibras por hectare do que o algodão ou linho, podendo ser, também, misturada a outras fibras orgânicas (Honório et al., 2006, p. 320).

No Brasil, seu cultivo e uso para fins de abuso não são permitidos, apenas no âmbito medicinal e sob algumas garantias legais. Já países como Canadá, Chile, Uruguai, Colômbia, Equador, México, Holanda, Itália e vários outros países da Europa, além de estados detentores de suas próprias leis, nos Estados Unidos, permitem o uso medicinal, assim como recreativo, desde que respeitadas as quantidades máximas estabelecidas por seus respectivos governos (Ferreira, 2017, p. 433).

Tanto seu uso terapêutico, assim como recreativo, por décadas tem sido motivo de inúmeros discursos e polêmicas, visto que a garantia e o direito individual das pessoas para plantar e consumir tem recaído sobre os interesses do estado, médicos e autoridades gorvernamentais, baseando-se em evidências clínicas e sociais controversas que apontam seus malefícios no âmbito da descriminalização (Pamplona, F A \& Malcher-Lopes, R, 2014).

Trazendo para o contexto nacional, segundo um levantamento realizado no ano de 2012, por volta de 1 milhão e meio de pessoas faziam o uso diário da planta, representando a droga ilícita de escolha no país (Lenard II, 2012). Outro fator a ser levado em consideração é a falta de estrutura para o tratamento de dependentes químicos no país. Sendo assim, partindo do pressuposto de que com a liberação do uso da planta poderia haver uma elevação no número de dependentes, este não seria a mais viável das escolhas, uma vez que oneraria ainda mais o serviço público de saúde, que já apresenta condições precárias para o tratamento de outras patologias (Araújo, 2014).

Já no âmbito medicinal, após o entendimento da sinalização molecular atuante na dor, analgesia, inflamação, apetite, motilidade gastrointestinal, ciclos do sono e atividades celulares, os mais de 55 fitocanabinoides extraídos e identificados passaram a representar uma enorme revolução na área terapêutica, sendo uma das grandes opções farmacológicas do século para o tratamento principal e adjuvante de patologias e transtornos neuropsiquiátricos decorrentes de diversas ações do sistema nervoso central (Grosso, 2020).

Dentre os diversos compostos, os dois mais importantes constituintes que atuam no sistema nervoso central são o 49-THC (delta 9- tetrahidrocanabinol), responsável pela sensação de agitação/euforia e delírio (quando inalada), enquanto que o CBD (Canabidiol) atua de maneira oposta, promovendo sensação de relaxamento, podendo ser extraído das sementes e raízes, apresentando uma maior concentração em folhas e flores (Campos et al., 2012, p. 3364).

No entanto, existem outros canabinoides isolados, pouco descritos, como Canabigerol (CBG), Canabinol (CBN) e o Canabicromeno (CBC). Apesar de apresentarem estruturas bioquímicas semelhantes, estes compostos podem manifestar 
diferentes atividades farmacológicas, a depender do tipo da interação com os componentes do sistema endocanabinoide, incluindo os receptores canabinóides e as enzimas de síntese de degradação de endocanabinoides (Ebling Flores \& Zamin, 2017, p. 224)

É sabido que o seu uso diário e prolongado por via inalátoria (fumada) está associado a efeitos danoso da cannabis sobre a função psicológica, incluindo um risco aumentado de desenvolvimento de quadros de psicose em indivíduos predispostos; alterações estruturais e funcionais do cérebro; e comprometimento cognitivo, principalmente em adolescentes com menos de 25 anos (Solowij et al., 2018, p. 32).

Estudos epidemiológicos abordaram doses orais de canabidiol- CBD da Cannabis sativa cronicamente consumida, constatando uma proteção contra os efeitos adversos do Tetrahidrocanabinol-THC inalado, incluindo sintomas psicóticos, desejo de consumo por outras drogas, perda de memória e perda de substância cinzenta do hipocampo, importante componente do sistema límbico (Morgan et al., 2010).

A administração por via oral de derivados isolados canabinoides em vivo demonstraram a perda e proteção contra restabelecimento do sentimento de medo. Entretanto, outros estudos investigaram principalmente os efeitos do THC, que reduziu as medidas fisiológicas do medo durante a extinção e recordação, mesmo tendo seu uso não recomendado para pacientes fóbicos, em rezão dos inúmeros efeitos psicoativos resultantes (de Vries et al., 2016, p. 533).

Assim, compreender os efeitos já descritos na literatura científica para compostos derivados da Cannabis, em especial a sativa, é fundamental para uma melhor argumentação embasada totalmente na ciência. Sendo assim, o presente estudo compila informações sobre os derivados canabinoides e os efeitos farmacológicos já descritos para os compostos canabidiol e delta-9-tetra-hidrocanabinol.

\section{Metodologia}

O presente estudo trata de uma revisão integrativa da literatura que reúne informações relevantes sobre uma temática de natureza teórica, exploratória e bibliográfica, com abordagem quanlitativa, utilizando-se das bases de dados: Medline, PubMed, Scielo, Periódicos Capes e LILACS, com auxílio dos seguintes descritores: canabinoides, cannabis sativa, canabidiol, delta 9 tetra-hidrocanabinol, patologia, sistema nervoso central.

A primeira fase do processo consistiu na elaboração de uma pergunta feita com base na estratégia PICO, utilizada para guiar a execução das outras atividades do processo e para facilitar a resolução da questão de pesquisa (Santos, Pimenta \& Nobre, 2007). Assim, foi formulado o seguinte questionamento: "Quais as aplicações nacionais e internacionais legais, toxicológicas e farmacoterapêuticas da Cannabis?"

A segunda fase, realizada entre maio a agosto de 2021, diz respeito a busca, seleção dos estudos e a avaliação, de acordo com os critérios de inclusão: artigos científicos originais, publicados em periódicos e indexados às bases de dados, escritos nos idiomas português e inglês, entre as datas de publicação de 2005 a 2021, que fossem trabalhos sobre características legais, toxicológicas e farmacoterapêuticas que envolvessem a Cannabis, além de estudos que tratassem sobre alguma dimensão das vantagens associadas ao uso da planta.

Na terceira etapa, após triagem inicial, apoiada nos critérios de inclusão e exclusão, verificou-se o título, resumo e palavras-chave dos resultados, dentro das próprias bases de dados. Logo, houve a seleção de 46 artigos, onde 5 (cinco) deles não abrangiam os critérios propostos em relação à temática do trabalho.

Por fim, foram lidos na íntegra 41 (quarenta e um) artigos elegíveis, e, então, avaliados criticamente. A análise dos dados ocorreu entre os meses de setembro e outubro de 2021.

Os estudos incluídos na presente revisão permitiu uma avaliação crítica do tema discutido, sendo possível identificar o vácuo do conhecimento, podendo direcionar futuras pesquisas complementares. 


\section{Resultados e Discussão}

\subsection{A Cannabis sativa e os Principais compostos Fitoquímicos}

A Cannabis sativa, em especial, sendo uma planta amplamente difundida no Brasil, conhecida popularmente como maconha, marijuana, charas, bhang, ganja e sinsemila, por apresentar em sua composição química uma grande quantidade de fitocanabinóides, tendo como princiapais compostos o Canabidiol- CBD e o delta 9- Tetra-hidrocanabinol, vem sendo utilizada na medicina na forma de fármacos, devido ao seu alto efeito em sistemas endógenos, tanto como tratamento principal, como adjuvante da epilepsia, ansiedade generalizada, ansiedade social, agitação psicomotora, trantorno obsessivo conpulsivo (TOC). Seus compostos também denotaram atividade analgésica, hipnótica, antipsicótica, neuroprotetora, antieméticas, antioxidantes, antiinflamatórias, antiartríticas e antineoplásicas (Brucki et al., 2015, p. 373).

Após a descoberta do sistema endocanabinóide, ocorrida na década de 60, a busca por seus respectivos ligantes iniciou-se. Os receptores CB1 (mais presentes no cérebro) e CB2 (mais encontrados em outras partes periféricas do corpo) representaram um avanço na ciência, assim como os compostos endógenos responsáveis por sua estimulação, a Anandamida e o 2-araquidonoilglicerol (Di Marzo et al., 2001, p. 348).

Observou-se que o sistema endocanabinóide seria responsável por regular diversas funções sinápticas e corporais periféricas, como balanço energético, sistema imunológico, controle emocional, aprendizagem e eventos aversivos aos humanos, como traumas e experiências nocivas, além do medo condicionado (Castillo et al., 2012, p. 77).

Em testes efetuados em laboratório evidenciou-se que os Canabidiol- CBD apresentou efeitos ansiolíticos quando administrado em doses mais baixas $(10 \mathrm{mg} / \mathrm{kg}$ ), enquanto que em dosagens mais altas (100 mg / kg) não observou-se uma ação calmante. Já por meio de outros ensaios realizados em camundongos, foi relatada a capacidade do CBD em inibir os efeitos causados pelas psicoses e ansiedades em voluntários sadios, utilizando $1 \mathrm{mg} / \mathrm{kg}$ de CBD por via oral, acompanhado por uma dose elevada de 0,5 mg/kg do delta 9-THC (Bitencourt et al., 2008, p. 854).

Figura 1. Estrutura química do Canabidiol (CBD).

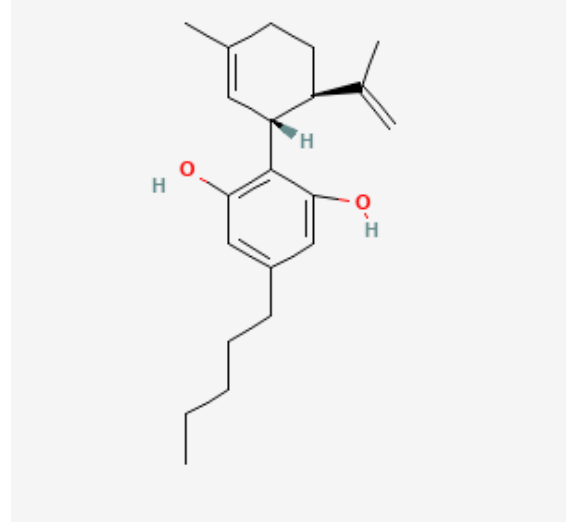

Fonte: https://pubchem.ncbi.nlm.nih.gov/compound/Cannabidiol.

Na Figura 1, de fórmula molecular $\mathrm{C}_{21} \mathrm{H}_{30} \mathrm{O}_{2}$, é importante observar a predominância de átomos de carbono e hidrogênio, o que conferem uma característica de lipossolubilidade à substância, responsável por sua facilidade de penetração pela barreira hematoencefálica (BHE).

Os resultados comprovaram a redução significativa da ansiedade e dos sintomas psicóticos desencadeados pelo delta 9-THC. Nos estudos clínicos psicológicos envolvendo humanos, o Canabidiol diminuiu intensamente a ansiedade ou o medo induzido experimentalmente. O mesmo demosntrou atividade ansiolítica associada a um teste simulado de falar em público 
em indivíduos saudáveis, assim como em indivíduos detentores de transtorno de ansiedade social-TAS, mostrando uma eficácia comparável à ipsapirona (um agonista 5-HT 1A R) e ao diazepam (Crippa et al., 2010, p. 562).

Já o delta 9-tetraidrocanabinol $\left(\Delta^{9}\right.$-THC) foi analisado e posteriormente recomendado por diversos autores por apresentar ação moduladora e estimulante sobre o apetite e manter a massa corporal dos indivíduos, principalmente aqueles portadores de algum tipo de câncer e portadores de HIV, assim como também para diminuir a sensação de náuseas e vômitos resultantes do tratamento com quimioterapia, além de atuar como analgésico de uso oral (Matos L. A. et al, 2017, p. 810).

Figura 2. Estrutura química do delta 9- Tetra-hidrocanabinol ( $\Delta 9-\mathrm{THC})$.

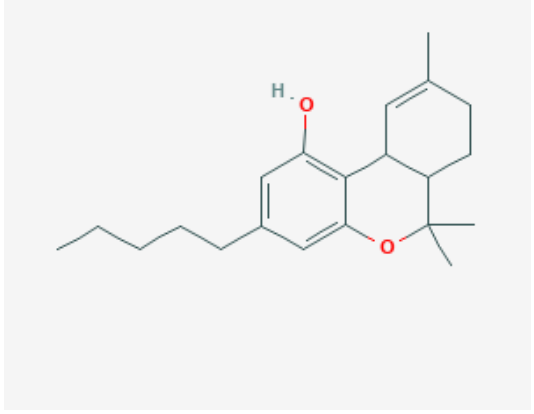

Fonte: https://pubchem.ncbi.nlm.nih.gov/compound/delta9-Tetrahydrocannabinol.

A Figura 2, destaca-se a presença de um anel aromático em sua estrutura molecular, o que também confere uma afinidade por lipídeos, em relação ao meio aquoso, contribuindo para sua passagem e acúmulo em tecidos ricos em gordura.

\subsection{Farmacocinética e Farmacodinâmica}

Os canabinoides aparesentam um alto perfil lipofílico, requerendo veículos especiais para facilitar sua dissolução em meio aquoso. O delta-9-tetrahidrocanabinol (THC), principal constituinde prasente na Cannabis sativa é um óleo viscoso volátil com alta solubilidade em lipídios que encontra-se como uma mistura de ácidos mono-carboxílicos, que facilmente sofre processo de descarboxilação após contato com temperaturas elevadas, sendo amplamente distribuído no tecido adiposo, baço e pulmões (Mechoulam, 2005, p. 914).

É metabolizado no fígado por hidroxilação microssomal e oxidação catalisada por enzimas do complexo do citocromo P450 (CYP), sendo também distribuído pelo cérebro, atingindo altas concentrações na área neocortical (córtex frontal), região límbica (hipocampo e amígdala), área sensorial (visual e auditória), área motora (gânglio basal e cerebelo) e ponte. Atravessa com rapidez a membrana alveolar, entrando no sangue pelos capilares pulmonares e daí levado rapidamente ao coração e bombeado diretamente ao encéfalo; desse modo, o pico de ação pode ser tão rápido quanto uma injeção venosa (Mercolini et al., 2008, p. 159).

Em relação a sua farmacodinâmica, são propostos dois tipos de mecanismos de interações. A primeira defende que sua ação é resultado de interações não específicas com as membranas celulares e organelas localizadas no cérebro, apoiando um mecanismo de perturbação da membrana. A segunda hipótese sugere uma interação e modulação específica com receptores do tipo canabinoide- CB1/ CB2. Definir um mecanismo específico é um desafio para os pesquisadores, já que em outros trabalhos foi demonstrado ação em alvos intracelulares, incluindo receptores de opióides e benzodiazepínicos, via de síntese de prostaglandinas, metabolismo de proteínas e ácidos nucléicos (Appendino et al., 2011). 
Já o Canabidiol (CBD) também apresenta caráter de solubilidade em gorduras, sendo amplamente distribuido por todo o organismo, passando pelo metabolismo de primeira passagem, onde o mesmo é biotransformado em diversos metabólitos ativos para o SNC, dentre eles: 7-hidroxi-CBD e ácido 7-oic-CBD, imitando a ação dos endocanabinoides, aumentando o seu efeito na regulação e transmissão de impulsos nervosos (Zhornitsky \& Potvin, 2012).

Age nos receptores CB1 no cérebro, que estão particularmente concentrados em regiões anatômicas associadas à cognição, memória, recompensa, ansiedade, percepção sensorial da dor, coordenação motora e função endócrina. Sugere-se que os receptores do tipo CB2, localizados mais perifericamente e alvo dessas moléculas, estejam conectados aos sistemas opioides, gabaérgicos, dopaminérgicos, noradrenérgicos, serotonérgicos, colinérgicos, glicocorticóides e prostaglandinas ((Pertwee, 2008).

Em estudos recentes in vitro constatou-se que o Canabidiol (CBD) inibia as enzimas do citocromo P450 o que determina uma potencial de interação farmacológica importante com outras susbtâncias. Outros trabalhos vem demonstrando a influência e importância dos receptores de serotonina, especialmente o 5HT-1 ${ }^{\mathrm{a}}$, um alvo ansiolítico já estabelecido pela literatura. Fármacos como a Buspirona e outros agonistas desse receptor tem sido utilizados no tratamento do medo, da ansiedade em geral e de outras doenças, sendo demonstrado através de estudos em vitro que o CBD age como ligante direto do mesmo, e em vivo, atuando como um modulador alostérico facilitando a sinalização do 5-HT 1A (Russo et al., 2005, p. 1040).

\subsection{Toxicidade}

Em ensaios de toxicidade aguda da Cannabis sativa (DL50), não demonstrou toxicidade aguda quando utilizado o óleo de CBD extraídos de folhas e flores, pois não obteve nenhum óbito após 24 horas de exposição , mesmo em concentrações elevadas . O perfil toxicológico da intoxicação por Cannabis Sativa L. está diretamente relacionada a relação de proporção entre a concentração do delta-9-tetraidrocanabinol e o canabidiol na amostra ingerida. Ambos compostos químicos apresentam elevada lipossolubilidade, o que faz com a que a absorção distribuição e metabolização ocorra rapidamente (Crippa et al., 2010).

Contudo, estudos pré-clínicos e clínicos também relatam efeitos adversos (AEs) e toxicidade após a ingestão de Canabidiol (CBD). Estudos publicados recentemente mostraram que em animais, os EAs de CBD incluíram toxicidade no desenvolvimento, mortalidade embriofetal, inibição e neurotoxicidade do sistema nervoso central, lesões hepatocelulares, redução da espermatogênese, alterações de peso de órgãos, alterações do sistema reprodutor masculino e hipotensão, embora em doses maiores do que as recomendadas para farmacoterapias humanas. Já em pesquisas envolvimendo humanos, o uso em doses elevadas para epilepsia e transtornos psiquiátricos resultaram em: anomalias hepáticas, interações com outros compostos farmacológicos, diarreia, fadiga, vômito e sonolência (Baker et al., 2010).

\subsection{Principais patologias com indicação de tratamento com Cannabis sativa}

Seus derivados, após extraídos e purificados são receitados para diversas patologias que envolvem a ansiedade excessiva como sintoma principal, em modelos de medo induzido em camundungos e humanos. Evidências pré-clínicas existentes apoim fortemente o CBD como um tratamento para transtorno de ansiedade generalizada, transtorno do pânico, transtorno de ansiedade social, transtorno obsessivo-compulsivo e transtorno de estresse pós-traumático. Outros estudos clínicos suportam um papel ansiolítico do CBD, mas atualmente está limitado à dosagem aguda (Blessing et al., 2015).

$\mathrm{O}$ uso dos endocanabinóides pode ser atribuído em várias patologias que acometem o sistema digestivo, influenciado os endocanabinóides na produção do ácido gástrico, náuseas, emese, sensação visceral, motilidade gastrointestinal, fibrinogénese hepática e inflamação intestinal. Em efeitos secundários da quimioterapia, como náuseas e vômitos pode ser usada como antagonista da dopamina análogos do THC- dronabinol e nabilona (Müller-Vahl \& Grotenhermen, 2013). 
Seu uso em condições médicas: dor crônica, dor neuropática e espasticidade resultante de esclerose múltipla foi evidenciado por meio de estudos pré-clínicos, demonstrando uma pequena janela terapêutica relacionada aos derivados da Cannabis sativa. Meta-análises de ensaios clínicos apontaram para uma promissora estratégia no uso dos mesmos, quando em comparação às moléculas sintéticas atualmente comercializadas (Hill et al., 2017).

Em relação ao câncer, esperimentos clínicos avaliaram o efeito do THC ou CBD no controle da dor relacionada ao mesmo com administração de doses variando de 2,7-43,2 mg / dia de THC e 0-40 mg / dia de CBD. Alguns efeitos colaterais relatados incluem sonolência, hipotensão, turvação mental, náuseas e vômitos. Há evidências que sugerem que a cannabis medicinal reduz a dor crônica ou neuropática em pacientes com câncer avançado, requerindo um número maior de estudos clínicos que abordem apenas essa temática (Blake et al., 2017).

Tabela 1. Derivados da Cannabis e suas respectivas aplicações farmacoterapêuticas.

\begin{tabular}{|c|c|c|}
\hline DERIVADO & EMPREGO & AUTOR \\
\hline Canabidiol - CBD & TAG- Transtorno de Ansiedade & Blessing et al., 2015. \\
\hline Delta-9- tetrahidrocanabinol- THC & Goneralizada & Hill et al., 2017. \\
\hline Canabidiol - CBD & Transtorno do pânico & Blessing et al., 2015. \\
\hline Análogos do delta 9 -THC & Náuseas & Müller-Vahl \& Grotenhermen, 2013. \\
\hline Canabidiol - CBD & TOG- Transtorno Obsessivo- & Blessing et al., 2015. \\
\hline $\begin{array}{c}\text { Delta- 9 Tetra-hidrocanabinol -THC } \\
\text { ou Canabidiol- CBD }\end{array}$ & Compulsivo & \\
\hline Canabidiol- CBD & Síndromes epilépticas refratárias & Huestis et al., 2019. \\
& infantis & \\
\hline
\end{tabular}

Fonte: Autores.

\subsection{Medicamentos presentes no mercado a base de Cannabis sativa}

Atualmente no mercado farmacêutico os medicamentos indicados a base de cannabis sativa estão relacionados ao tratamento da dor, fabricados nas formas famacêuticas de spray, óleo ou aerossol, como o da empresa Bedrocan®, que comercializa uma variação da Cannabis sativa contendo 22\% de THC e um nível de CBD inferior a 1\%. É o produto medicinal de cannabis mais amplamente difundido, estando entre os mais esolhidos para pesquisas do que outros produtos (Productos Bedrocan produce cinco cepas de cannabis medicinal, n.d.).

Vários estudos pré-clínicos e clínicos levaram à aprovação da FDA do Epidiolex®, um medicamento CBD purificado formulado para administração oral para o tratamento de síndromes epilépticas refratárias infantis, pela Food and Drug Administration dos EUA em 2018. (Huestis et al., 2019).

O Sativex ${ }^{\circledR}$, composto por delta-9-tetrahidrocanabinol e o canabidiol que atuam diretamente no sistema nervoso, levando ao relaxamento dos músculos e alívio da rigidez e dor muscular. É produzido pela GW Pharmaceuticals a partir de Cannabis, sendo lançado em 2005 no Canadá, prescrito para o alívio da rigidez muscular associada à esclerose múltipla. (Sativex - Bula de Sativex, n.d.). Já no Brasil existe um outro medicamento com as mesmas características chamado Mevatyl® (tetraidrocanabinol (THC), $27 \mathrm{mg} / \mathrm{mL}$ + canabidiol (CBD), $25 \mathrm{mg} / \mathrm{mL}$ ), sendo o primeiro medicamento com registro aprovado pela Agência Nacional de Vigilância Sanitária (Anvisa), e desenvolvido por meio de canabinoides obtidos a partir da Cannabis sativa, na forma farmacêutica solução oral- spray (Mevatyl® - CRF-SP - Conselho Regional de Farmácia do Estado de São 
Paulo, 2017).

Nos Estados Unidos o Dronabinol $®$, uma forma sintética do- $\Delta 9$-THC, teve seu resgistro aprovado em 1986 pelo FDA para tratamento antiemético, usado durante a quimioterapia e estimulante do apetite em pacientes portadores de AIDS com perda de peso (Grotenhermen \& Müller-Vahl, 2012).

Outro medicamento é o Cannador®, vendi na forma de cápsula oral contendo um extrato de planta inteira, com conteúdo de THC padronizado e uma quantidade de CBD controlada para ficar dentro de uma faixa estreita fixa com uma razão THC: CBD de cerca de 2: 1. Foi usado em vários ensaios clínicos e é uma marca registrada em muitos países. Foi clinicamente testado para redução da rigidez muscular, espasmos e dor associada na esclerose múltipla, para anorexia / caquexia em pacientes com câncer e para o controle da dor pós-operatória (International Association for Cannabis as Medicine, n.d.).

O Cesamet®, fabricado pelo laboratório Meda, é canabinoide sintético do tetraidrocanabinol (THC) agindo como agonista de receptores $\mathrm{CB} 1$, indicado quando os pacientes não respondem aos antiemeticos convencionais e como analgésico auxiliar para dor neuropática (Souza, Y P, 2017).

\subsection{Perspectivas para o desenvolvimento de medicamentos a base da Cannabis sativa}

A partir de outros testes, foi descrito que pequenas doses do THC diminuem a concentração da proteína betaamiloides que é responsável pelo Alzheimer, indicando uma prerspectiva promissora no desenvolvimento de produtos que apresentem uma maior funcionalidade que os medicamentos convencionais (Baptista, 2014).

Já no pakinson, estudos mostram que $75 \mathrm{mg} /$ dia ou 300mg/dia de canabidiol puro resulta em uma eficiente diminuição nos sintomas e também proporciona uma melhor qualidade de vida ao usuário portador (Brucki et al., 2015).

Assim, a indústria farmacêutica tem dado cada vez mais atenção ao desenvolvimento de produtos contendo derivados da Cannabis sativa, tendo a opção de realizar mudanças químicas em suas moléculas, expandindo a sua aplicabilidade para outras doenças, além de reduzir efeitos adversos resultantes do uso de tais compostos. Por se trata de um método custoso, a extração e purificação ainda gera um alto custo para as empresas, o que tende a mudar com o avanço industrial tecnológico e popularização de outros medicamentos que se utilizam da planta.

\subsection{Legislação brasileira sobre a Cannabis sativa}

Nacionalmente, a lei antidrogas 11.343/2006 dispões sobre as punições para o tráfico e o suo de drogas, definidas como substâncias ou produtos capazes de causar de pendência. Assim, vender, produzir e portar drogas, em qualquer que seja sua quantidade, era considerado crime. Em geral, quaisquer atividades que estejam ligadas a produção e à distribuição não medicinal eram punidas com prisão. Seguindo outros países, como Argentina, que recentemente liberou o autocultivo de Cannabis sativa $L$ para uso medicinal e permitiiu a venda de óleos, cremes e outros derivados da planta em farmácias autorizadas, segundo a nova regulamentação publicada em boletim oficial, o país passou a debater e facilitar o acesso a produtos derivados da Cannabis sativa (Ferreira, 2017).

O uso no Brasil de derivados da Cannabis só pode ser feito legalmente através da compra do produto, importação ou autorização judicial prévia para extração, além de uso medicinal e não recreativo, limitada a casos específicos. A RDC 327 de 2019 foi um marco frente ao acesso de produtos derivados da Cannabis Saativa, pois regulamentou a autorização sanitária para a fabricação e importação, comercialização, prescrição, a dispensação, o monitoramento e a fiscalização de produtos a base de Cannabis para fins medicinais (Órgão: Ministério da Saúde/Agência Nacional de Vigilância Sanitária/Diretoria Colegiada, 2019).

Tal burocracia é expressa no enorme número ações judiciais iniciadas por familiares de pacientes portadores, 
principalmente de quadros epilépticos, para autorização de compra pelo Sistema único de Saúde ou o direto de plantar e extrair as susbstâncias de interesse farmacológico. Dentre os projetos apresentados no senado estão o Projeto de Lei número 514/2017; 5.295/2019; 4.776/2019; 5.158/2019, buscando deburocrtizar o acesso a esses fitocanabinoides (Cannabis medicinal: realidade à espera de regulamentação, n.d.)

Recentemente, a Sexta Turma do Superior Tribunal de Justiça (STJ) firmou o entendimento de que a posse de objeto para cultivar Cannabis não pode ser enquadrada no Artigo 34 da Lei de Drogas, que prevê pena de três a dez anos de reclusão para esse tipo de crime, se o plantio for destinado exclusivamente para o consumo próprio. Com esse entendimento, os ministros do STJ concederam um habeas corpus para garantir que um homem flagrado com 5,8 gramas de haxixe e oito plantas de Cannabis não seja processado pelo Artigo 34 da Lei de Drogas, já que em sua casa foram encontrados também diversos materiais para o cultivo e extração de óleo da planta. Tal dispositivo, contudo, só pode ser aplicado na hipótese em que a produção da droga seja destinada ao narcotráfico- artigo 33 da Lei de Drogas (Ter item de cultivo de Cannabis para uso pessoal não justifica ação, 2021a).

Também em 2021 foi aprovada a RDC 473, que enquadra a Cannabis sativa na lista de substancias de controle especial- portaria 344/98, ficando sujeitos aos controles referentes a esta Lista os medicamentos registrados na Anvisa que possuam em sua formulação derivados de Cannabis sativa, em concentração de no máximo 30 mg de tetrahidrocannabinol (THC) por mililitro e $30 \mathrm{mg}$ de canabidiol por mililitro, permitando a importação de Produto derivado de Cannabis, por pessoa física, para uso próprio, mediante prescrição de profissional legalmente habilitado (RESOLUÇÃO DE DIRETORIA COLEGIADA - RDC N 473, DE 24 DE FEVEREIRO DE 2021 - RESOLUÇÃO DE DIRETORIA COLEGIADA - RDC Nº 473, DE 24 DE FEVEREIRO DE 2021 - DOU - Imprensa Nacional, 2021).

Por fim, em junho do mesmo ano, a comissão especial da Câmara dos Deputados aprovou o projeto de Lei 399/15, que legaliza o cultivo da Cannabis sativa no Brasil para fins medicinais, veterinários, científicos e industriais (Comissão aprova proposta para legalizar no Brasil o cultivo de Cannabis para fins medicinais Fonte: Agência Câmara de Notícias, n.d.).

\section{Conclusão}

Com isso, podemos afirmar que o uso do Canabodiol- CBD em humanos é fortemente apoiado como uma alternativa para diversas patologias que compartilham de sintomas de ansiedade e medo, com dosagens que podem variar de 300 a 600 mg. Mais estudos são necessários para estabelecer se a dosagem crônica também é responsável por efeitos ansiolíticos potentes em humanos (Blessing et al., 2015, p. 832).

Esses achados representam uma alternativa de tratamento para pacientes com doenças, nas quais apresentam como característica fundamental uma falha na extinção das memórias de medo que contribuem para o desenvolvimento e a persistência do trauma, acarretando uma resposta patológica condicionada à sensação desagradável desecadeada pela sensação de perigo de forma prolongada (Solowij et al., 2018, p. 31).

Os canabinoides podem assumir um papel de adjuvante em terapias para transtornos de ansiedade, uma vez que indivíduos saudáveis ao receber THC mostraram significativo aprimoramento da memória de extinção devido a ativação do córtex pré-frontal e hipocampo ventromedial. Essas estruturas modulam os chamados circuitos pré-frontal-límbicos importantes para a extinção do medo. O THC também demonstrou efeitos sinérgicos com o CBD sobre a conectividade funcional entre o núcleo basolateral da amígdala e o córtex pré-frontal em situações de ameaça socioemocional, reduzindo a percepção dessas (Korem et al., 2016).

Assim, conclui-se que o isolamento de ligantes endógenos (endocanabinoides, principalmente anandamida e 2araquidonoilglicerol) demonstram que os canabinoides podem apresentar uma função significativa na mediação de diversos processos neurofisiológicos, inclusive na nocicepção. O uso da Cannabis sativa como fonte vegetal de moléculas tem 
demonstrado efeitos satisfatórios, tanto nos testes em vitro, assim como em seres humano, sendo sua ação comparada, e até superada à de medicamentos benzodizepínicos, antidepressivos tricíclicos, inibidores seletivos da recaptação de serotonina, anticonvulsivantes, antipsicóticos e anti-inflamatórios.

Com uma pespectiva a longo prazo no Brasil e no mundo, o uso de tais compostos na produção de medicamentos pelo setor industrial farmacêtuco tende a se tornar mais barato e acessível à população, tendo em vista que se trata de uma planta de fácil cultivo e que apresenta grande número de estudos científicos atestando e reforçando sua segurança. Contudo, a aplicação futura de seus derivados em sistemas públicos de saúde pelo mundo pode sofrer considerável resistência, pelo fato de se tratar de uma planta rotineiramente associada à violência e o crime organizado.

\section{Agradecimentos}

Mesmo com todas as dificuldades, que por muitas vezes nos fez pensar em desistir, ao olharmos para trás podemos ver como grande foi nossa jornada e, hoje finalizamos um ciclo e iniciamos outro. Assim, agradeço a Deus por sempre estar conosco e prover forças para enfrentar e guiar por caminhos melhores. Agradeço à minha família, aos meus pais por serem sempre meu alicerce, em especial à minha mãe. Agradeço as amizades que adquiri ao longo de toda graduação, aos meus professores por toda paciência e por todo conhecimento e ensinamentos repassados, garantindo e oferecendo sempre o melhor. Hoje podemos dizer: nós conseguimos! Agradecemos ao leitor desse trabalho, por se tratar de um tema tão relevante, que ao longo dos anos despertou tanta curiosidade no meio acadêmico/científico.

\section{Referências}

Appendino, G., Chianese, G., \& Taglialatela-Scafati, O. (2011). Cannabinoids: Occurrence and Medicinal Chemistry. Current Medicinal Chemistry, 18(7), 1085-1099.

Araújo, T. Almanaque das Drogas. São Paulo: Leya, 2014.

Baptista, Lucas. (2014). Uso de maconha para o retardo dos efeitos do Alzheimer.

Baker, T. A., Bufalino, M. E., Ford, M. L., \& Kale, S. R. (2010). Five Years of Experience with the ConnectOregon Multimodal Funding Program. Transportation Research Record: Journal of the Transportation Research Board, 2174(1), 68-76. https://doi.org/10.3141/2174-10

Blake, A., Wan, B. A., Malek, L., DeAngelis, C., Diaz, P., Lao, N., Chow, E., \& O’Hearn, S. (2017). A selective review of medical cannabis in cancer pain management. Annals of Palliative Medicine, 6(S2), S215-S222. https://doi.org/10.21037/apm.2017.08.05

Blessing, E. M., Steenkamp, M. M., Manzanares, J., \& Marmar, C. R. (2015). Cannabidiol as a Potential Treatment for Anxiety Disorders. Neurotherapeutics, 12(4), 825-836. https://doi.org/10.1007/s13311-015-0387-1

Bitencourt, R. M., Pamplona, F. A., \& Takahashi, R. N. (2008). Facilitation of contextual fear memory extinction and anti-anxiogenic effects of AM404 and cannabidiol in conditioned rats. European Neuropsychopharmacology, 18(12), 849-859. https://doi.org/10.1016/j.euroneuro.2008.07.001

Brucki, S. M. D., Frota, N. A., Schestatsky, P., Souza, A. H., Carvalho, V. N., Manreza, M. L. G., Mendes, M. F., Comini-Frota, E., Vasconcelos, C., Tumas, V., Ferraz, H. B., Barbosa, E., \& Jurno, M. E. (2015). Cannabinoids in neurology - Brazilian Academy of Neurology. Arquivos de Neuro-Psiquiatria, 73(4), 371-374. https://doi.org/10.1590/0004-282x20150041

Campos, A. C., Moreira, F. A., Gomes, F. V., Del Bel, E. A., \& Guimarães, F. S. (2012). Multiple mechanisms involved in the large-spectrum therapeutic potential of cannabidiol in psychiatric disorders. Philosophical Transactions of the Royal Society B: Biological Sciences, 367(1607), 3364-3378. https://doi.org/10.1098/rstb.2011.0389

Castillo, P. E., Younts, T. J., Chávez, A. E., \& Hashimotodani, Y. (2012). Endocannabinoid Signaling and Synaptic Function. Neuron, 76(1), 70-81. https://doi.org/10.1016/j.neuron.2012.09.020

Cannabis medicinal: realidade à espera de regulamentação. (n.d.). Senado Federal. https://www12.senado.leg.br/noticias/infomaterias/2021/07/cannabismedicinal-realidade-a-espera-de-regulamentacao

Comissão aprova proposta para legalizar no Brasil o cultivo de Cannabis sativa para fins medicinais Fonte: Agência Câmara de Notícias. (n.d.). camara dos deputados. https://www.camara.leg.br/noticias/769630-comissao-aprova-proposta-para-legalizar-no-brasil-o-cultivo-de-cannabis-sativa-para-fins-medicinais

Crippa, J. A. S., Zuardi, A. W., \& Hallak, J. E. C. (2010). Uso terapêutico dos canabinoides em psiquiatria. Revista Brasileira de Psiquiatria, 32(suppl 1), 556-566. https://doi.org/10.1590/s1516-44462010000500009 
Vries, M., Van Rijckevorsel, D. C. M., Vissers, K. C. P., Wilder-Smith, O. H. G., \& Van Goor, H. (2016). Single dose delta-9-tetrahydrocannabinol in chronic pancreatitis patients: analgesic efficacy, pharmacokinetics and tolerability. British Journal of Clinical Pharmacology, 81(3), 525-537. https://doi.org/10.1111/bcp.12811

Di -Marzo, V., Bisogno, T., \& De Petrocellis, L. (2001). Anandamide: some like it hot. Trends in Pharmacological Sciences, 22(7), 346-349. https://doi.org/10.1016/s0165-6147(00)01712-0

Ebling- Flores, L., \& Zamin, L. L. (2017). Potencial neuroprotetor, antioxidante e anti-inflamatório do Canabidiol: relevância e perspectivas para o tratamento de doenças neurodegenerativas. Revista de Ciências Médicas e Biológicas, 16(2), 224. https://doi.org/10.9771/cmbio.v16i2.20568

Ferreira, S. (2017). Liberação da maconha. Revista Bioética, 25(3), 431-436. https://doi.org/10.1590/1983-80422017253000

Grosso, Adriana F. (2020). Cannabis: de planta condenada pelo preconceito a uma das grandes opções terapêuticas do século. Journal of Human Growth and Development, 30(1), 94-97. https://dx.doi.org/10.7322/jhgd.v30.9977

Grotenhermen, F., \& Müller-Vahl, K. (2012). The Therapeutic Potential of Cannabis and Cannabinoids. Deutsches Aerzteblatt Online. https://doi.org/10.3238/arztebl.2012.0495

Hill, K. P., Palastro, M. D., Johnson, B., \& Ditre, J. W. (2017). Cannabis and Pain: A Clinical Review. Cannabis and Cannabinoid Research, 2(1), 96-104. https://doi.org/10.1089/can.2017.0017

Honório, K. M., Arroio, A., \& Silva, A. B. F. d. (2006). Aspectos terapêuticos de compostos da planta Cannabis sativa. Química Nova, 29(2), 318-325. https://doi.org/10.1590/s0100-40422006000200024

Huestis, M. A., Solimini, R., Pichini, S., Pacifici, R., Carlier, J., \& Busardò, F. P. (2019). Cannabidiol Adverse Effects and Toxicity. Current Neuropharmacology, 17(10), 974-989. https://doi.org/10.2174/1570159x17666190603171901

International Association for Cannabis as Medicine. (n.d.). cannabis medicine. med.org/index.php?tpl=def\&amp;id=241\&amp;lng=en\&amp;red=deflist

Lenard II (2012). Levantamento Nacional de Álcool e Drogas. São Paulo (BR): Instituto Nacional de Ciência e Tecnologia para Políticas Públicas do Álcool $e$ Outras Drogas, Universidade Federal de São Paulo [PDF file]. Disponível em: https://inpad.org.br/wp-content/uploads/2014/03/Lenad-IIRelat\%C3\%B3rio.pdf

Matos L. A. et al, R. (2017). O Uso do Canabidiol no Tratamento da Epilepsia. revista virtual de quimica, 9(2), 786-814.

Mechoulam, R. (2005). Plant cannabinoids: a neglected pharmacological treasure trove. British Journal of Pharmacology, 146(7), 913-915. https://doi.org/10.1038/sj.bjp.0706415

Mercolini, L., Musenga, A., Comin, I., Baccini, C., Conti, M., \& Raggi, M. A. (2008). Determination of plasma and urine levels of $\Delta 9$-tetrahydrocannabinol and its main metabolite by liquid chromatography after solid-phase extraction. Journal of Pharmaceutical and Biomedical Analysis, 47(1), 156-163. https://doi.org/10.1016/j.jpba.2007.12.023

Mevatyl® - CRF-SP - Conselho Regional de Farmácia do Estado de São Paulo. (2017, 16 de janeiro). Home - CRF-SP - Conselho Regional de Farmácia do Estado de São Paulo. http://www.crfsp.org.br/noticias/8277-mevatyl.html

Morgan, C. J., Freeman, T. P., Schafer, G. L., \& Curran, H. V. (2010b). Cannabidiol Attenuates the Appetitive Effects of $\Delta 9$-Tetrahydrocannabinol in Humans Smoking Their Chosen Cannabis. Neuropsychopharmacology, 35(9), 1879-1885. https://doi.org/10.1038/npp.2010.58

Müller-Vahl, K., \& Grotenhermen, F. (2013). Cannabis Therapy. Deutsches Aerzteblatt Online. https://doi.org/10.3238/arztebl.2013.0144a

Pamplona, F. A. Malcher-Lopes, R. (2014). O ano da mudança. Revista da Biologia, São Paulo, 13(1).

Pertwee, R. G. (2008). The diverse CB1 and CB2 receptor pharmacology of three plant cannabinoids: $\Delta 9$-tetrahydrocannabinol, cannabidiol and $\Delta 9$ tetrahydrocannabivarin. British Journal of Pharmacology, 153(2), 199-215. https://doi.org/10.1038/sj.bjp.0707442

Productos - Bedrocan produce cinco cepas de cannabis medicinal. (n.d.). Bedrocan - beyond pioneering. https://bedrocan.com/es/productos/

Resolução de diretoria colegiada - rdc no 473, de 24 de fevereiro de 2021 - resolução de diretoria colegiada - rdc $n^{\circ} 473$, de 24 de fevereiro de 2021 - dou $\begin{array}{lllllll}\text { Imprensa Nacional. } & \text { (2021, } & 3 & \text { de março). } & \text { Home }\end{array}$ http://antigo.anvisa.gov.br/documents/10181/6236630/\%282\%29RDC_473_2021_.pdf/7a65445f-52a1-4533-97c7-6d96eff3b8e1

Russo, E. B., Burnett, A., Hall, B., \& Parker, K. K. (2005). Agonistic Properties of Cannabidiol at 5-HT1a Receptors. Neurochemical Research, 30(8), 10371043. https://doi.org/10.1007/s11064-005-6978-1

Sativex - Bula de Sativex. (n.d.). Bulário. https://www.bulario.com/sativex/

Santos, C. M. C., Pimenta, C. A. M. \& Nobre, M. R. C. (2007). A estratégia PICO para a construção da pergunta de pesquisa e busca de evidências. Revista Latino-Americana de Enfermagem, 15(3), 508-511. https://doi.org/10.1590/S0104-11692007000300023.

Solowij, N., Broyd, S. J., Beale, C., Prick, J.-A., Greenwood, L.-m., van Hell, H., Suo, C., Galettis, P., Pai, N., Fu, S., Croft, R. J., Martin, J. H., \& Yücel, M. (2018). Therapeutic Effects of Prolonged Cannabidiol Treatment on Psychological Symptoms and Cognitive Function in Regular Cannabis Users: A Pragmatic Open-Label Clinical Trial. Cannabis and Cannabinoid Research, 3(1), 21-34. https://doi.org/10.1089/can.2017.0043

Souza, Y. P. (2017). Sínteses e aplicações recentes do $\Delta 9$-tetraidrocanabinol (thc) e seus derivados em química medicinal. 
Research, Society and Development, v. 10, n. 15, e18101522408, 2021

(CC BY 4.0) | ISSN 2525-3409 | DOI: http://dx.doi.org/10.33448/rsd-v10i15.22408

Ter item de cultivo de maconha para uso pessoal não justifica ação. (2021a, 22 de setembro). Agência Brasil. https://agenciabrasil.ebc.com.br/justica/noticia/2021-09/justica/noticia/2021-09/ter-item-de-cultivo-de-maconha-para-uso-pessoal-nao-justifica-acao

Korem, N., Zer-Aviv, T. M., Ganon-Elazar, E., Abush, H., \& Akirav, I. (2016). Targeting the endocannabinoid system to treat anxiety-related disorders. Journal of Basic and Clinical Physiology and Pharmacology, 27(3). https://doi.org/10.1515/jbcpp-2015-0058

Zhornitsky, S., \& Potvin, S. (2012). Cannabidiol in Humans-The Quest for Therapeutic Targets. Pharmaceuticals, 5(5), 529-552. https://doi.org/10.3390/ph5050529 